\title{
Intermittent Preventive Treatment for Malaria in Papua New Guinean Infants Exposed to Plasmodium falciparum and P. vivax: A Randomized Controlled Trial
}

\author{
Nicolas Senn ${ }^{1,2,3,49}$, Patricia Rarau ${ }^{19}$, Danielle I. Stanisic ${ }^{1,5}$, Leanne Robinson ${ }^{1,5}$, Céline Barnadas ${ }^{1,5}$, \\ Doris Manong ', Mary Salib ', Jonah Iga ', Nandao Tarongka', Serej Ley', Anna Rosanas-Urgell', \\ John J. Aponte ${ }^{6}$, Peter A. Zimmerman ${ }^{7}$, James G. Beeson ${ }^{5,8}$, Louis Schofield ${ }^{5}$, Peter Siba', \\ Stephen J. Rogerson ${ }^{2}$, John C. Reeder ${ }^{8}$, Ivo Mueller ${ }^{1,5,6 *}$
}

1 Papua New Guinea Institute of Medical Research, Madang, Papua New Guinea, 2 Department of Medicine, University of Melbourne, Melbourne Australia, 3 Swiss Tropical and Public Health Institute, Basel, Switzerland, $\mathbf{4}$ University of Basel, Basel, Switzerland, $\mathbf{5}$ Infection and Immunity Division, Walter and Eliza Hall Institute, Melbourne, Australia, 6 Barcelona Centre for International Health Research (CRESIB), Barcelona, Spain, 7 Center for Global Health and Diseases, Case Western Reserve University, Cleveland, Ohio, United States of America, 8 Burnet Institute, Melbourne, Australia

\begin{abstract}
Background: Intermittent preventive treatment in infants (IPTi) has been shown in randomized trials to reduce malariarelated morbidity in African infants living in areas of high Plasmodium falciparum (Pf) transmission. It remains unclear whether IPTi is an appropriate prevention strategy in non-African settings or those co-endemic for $P$. vivax (PV).

Methods and Findings: In this study, 1,121 Papua New Guinean infants were enrolled into a three-arm placebo-controlled randomized trial and assigned to sulfadoxine-pyrimethamine (SP) (25 mg/kg and $1.25 \mathrm{mg} / \mathrm{kg}$ ) plus amodiaquine (AQ) (10 mg/kg, $3 \mathrm{~d}, n=374)$, SP plus artesunate (AS) $(4 \mathrm{mg} / \mathrm{kg}, 3 \mathrm{~d}, n=374)$, or placebo $(n=373)$, given at $3,6,9$ and $12 \mathrm{mo}$. Both participants and study teams were blinded to treatment allocation. The primary end point was protective efficacy (PE) against all episodes of clinical malaria from 3 to 15 mo of age. Analysis was by modified intention to treat. The PE (compared to placebo) against clinical malaria episodes (caused by all species) was $29 \%(95 \% \mathrm{Cl}, 10-43, p \leq 0.001)$ in children receiving SP-AQ and $12 \%(95 \% \mathrm{Cl},-11$ to $30, p=0.12)$ in those receiving SP-AS. Efficacy was higher against $P f$ than $P v$. In the SP-AQ group, $P f$ incidence was $35 \%(95 \% \mathrm{Cl}, 9-54, p=0.012)$ and $P v$ incidence was $23 \%(95 \% \mathrm{Cl}, 0-41, p=0.048)$ lower than in the placebo group. IPTi with SP-AS protected only against $P f$ episodes (PE $=31 \%, 95 \% \mathrm{Cl}, 4-51, p=0.027)$, not against $P V$ episodes $(\mathrm{PE}=6 \%, 95 \% \mathrm{Cl},-24$ to $26, p=0.759)$. Number of observed adverse events/serious adverse events did not differ between treatment arms $(p>0.55)$. None of the serious adverse events were thought to be treatment-related, and the vomiting rate was low in both treatment groups (1.4\%-2.0\%). No rebound in malaria morbidity was observed for 6 mo following the intervention.
\end{abstract}

Conclusions: IPTi using a long half-life drug combination is efficacious for the prevention of malaria and anemia in infants living in a region highly endemic for both $P f$ and $P V$.

\section{Trial registration: ClinicalTrials.gov NCT00285662}

Please see later in the article for the Editors' Summary.

Citation: Senn N, Rarau P, Stanisic DI, Robinson L, Barnadas C, et al. (2012) Intermittent Preventive Treatment for Malaria in Papua New Guinean Infants Exposed to Plasmodium falciparum and P. vivax: A Randomized Controlled Trial. PLoS Med 9(3): e1001195. doi:10.1371/journal.pmed.1001195

Academic Editor: Sanjeev Krishna, St George's Hospital Medical School, United Kingdom

Received July 5, 2011; Accepted February 9, 2012; Published March 27, 2012

Copyright: (c) 2012 Senn et al. This is an open-access article distributed under the terms of the Creative Commons Attribution License, which permits unrestricted use, distribution, and reproduction in any medium, provided the original author and source are credited.

Funding: This work was supported by a grant to the PNG Institute of Medical Research from the Bill \& Melinda Gates Foundation's Global Health Program (Grand ID\# 34678). The funder had no role in study design, data collection and analysis, decision to publish, or preparation of the manuscript. This work was made possible through Victorian State Government Operational Infrastructure Support and Australian Government NHMRC IRIISS.

Competing Interests: SJR is a member of the PLOS Medicine Editorial Board. The authors have declared that no competing interests exist.

Abbreviations: $A E$, adverse event; $A Q$, amodiaquine; $A S$, artesunate; $A T P$, according to protocol; EPI, Expanded Programme on Immunization; $\mathrm{Hb}$, hemoglobin IPT, intermittent preventive treatment; IPTi, intermittent preventive treatment in infants; IRR, incidence rate ratio; LDR-FMA, ligase detection reaction/fluorescent microsphere assay; mITT, modified intention to treat; PE, protective efficacy; Pf, Plasmodium falciparum; PNG, Papua New Guinea; Pv, Plasmodium vivax; PYAR, person-year at risk; $\mathrm{SAE}$, serious adverse event; $\mathrm{SP}$, sulfadoxine-pyrimethamine

* E-mail: ivomueller@fastmail.fm

9 These authors contributed equally to this work. 


\section{Introduction}

Malaria and anemia are major causes of morbidity and mortality in children in tropical countries [1]. Several preventive strategies have proven to be effective in reducing this burden, such as insecticide-treated bed nets [2], indoor residual spraying [3], or prompt diagnosis and treatment of malaria with artemisinin combination therapy $[1,4]$. Additionally, the preventative administration of a full treatment course of antimalarial drugs at fixed intervals regardless of illness episodes, named intermittent preventive treatment (IPT), has been shown to reduce malariarelated morbidity in different high risk groups $[5,6]$. This strategy is increasingly widely used in pregnant women (IPTp) $[7,8]$, with a positive effect on antenatal parasitemia, placental malaria, maternal hemoglobin $(\mathrm{Hb})$, birth weights, and newborn malaria infections [7,9]. Several studies have shown that IPT is effective in reducing malaria incidence when given to infants at the time of routine immunizations (IPTi), or to older children in areas where malaria transmission is highly seasonal (IPTc) [10-13].

In the past $10 \mathrm{y}$, eight randomized controlled trials have investigated IPTi in different African countries, with various drug regimens. Sulfadoxine-pyrimethamine (SP), dispensed as a single dose three or four times during the first year of life, is the most studied drug and has been associated with a protective efficacy (PE) against clinical malaria episodes of 30\% (ranging from 20\% to 59\%) in populations where SP resistance is not extensive. It also reduced the risk of anemia by $21 \%$ in these trials [10]. In all but one trial the preventative effect of the IPTi dose was restricted almost exclusively to the first $5 \mathrm{wk}$ after an individual IPTi dose, with little effect thereafter [10]. While IPTi with SP retains efficacy in areas with moderate SP resistance [14,15], SP has failed to prevent malaria in areas with very high resistance levels [16,17]. Based on this evidence, the World Health Organization recommends IPTi as a suitable malaria control intervention in areas of high malaria burden and low-to-moderate SP resistance [18].

To date, all reported IPTi studies have been carried out exclusively in sub-Saharan Africa in populations where Plasmodium falciparum $(P f)$ is the predominant parasite and $P$. vivax $(P v)$ is uncommon because of the high prevalence of Duffy negativity [19]. However, in highly endemic areas of the Southwest Pacific [20,21], Southeast Asia [22], the Americas [23], and the horn of Africa [24], $P v$ is a major cause of malaria morbidity, including severe illness and death in young children in particular. Therefore, although IPTi may have significant benefits in these regions, to date there are no data of which we are aware on IPTi efficacy in non-African settings, or on the use of IPTi for malaria due to $P$. vivax. Results from Africa cannot be easily extrapolated to other settings because of differences in the biology of $P f$ and $P v$ [25], in particular the ability of $P v$ to relapse from long-lasting liver stages, and also the ability of $P v$ to quickly acquire resistance to SP [26].

In order to determine the efficacy of IPTi in reducing the burden of malaria (all cause as well as $P f$ and $P v$ specific) and anemia in a $P f$ and $P v$ co-endemic setting, we undertook a threearm randomized placebo-controlled trial of IPTi in an area of Papua New Guinea (PNG) that is highly endemic for both $P f$ and $P$. We tested two different drug regimens, combining a longacting drug (SP) with either $3 \mathrm{~d}$ of amodiaquine (AQ) (another long half-life drug) or $3 \mathrm{~d}$ of artesunate (AS) (a short half-life drug), given in conjunction with routine immunization activities. These two drug regimens were chosen for two reasons: (1) they were the first and second line treatments in PNG at the time of the commencement of the study, facilitating their possible implementation into PNG standard practice as treatments for IPTi, and (2) the comparison allows differentiation between the importance of potentiallyimproved clearance of existing infections (using SP-AS) versus better prevention of new infections post-treatment (using the long-acting combination of SP-AQ).

\section{Methods}

\section{Trial Design, Sites, and Study Participants}

We undertook an individually randomized placebo-controlled trial of two different drug regimens (SP [single dose] in combination with either $3 \mathrm{~d}$ of AQ [SP-AQ] or $3 \mathrm{~d}$ of AS [SPAS]) given as IPTi at routine vaccination time points $(3,6$, and $9 \mathrm{mo}$ ) and at $12 \mathrm{mo}$ in conjunction with routine vitamin A supplementation as part of the World Health Organization's Expanded Programme on Immunization (EPI) in PNG. Participants, field teams, and investigators were all blinded with respect to treatment allocation. The efficacy of the intervention was assessed for a 12-mo period from enrollment to a primary assessment time point at 15 mo of age, with a 6 -mo extended follow-up (15-21 mo) to assess potential rebound.

The study was carried out between 6 June 2006 and 14 May 2010 on the north coast of PNG in the Mugil area of Sumkar District, Madang Province. The study site included 20 villages situated in an $\sim 400-\mathrm{km}^{2}$ coastal area $30-60 \mathrm{~km}$ north of Madang town. The region receives over $3,000 \mathrm{~mm}$ of rainfall annually, with a short dry season (June to October), and is considered to have hyper-endemic malaria $[27,28]$. Study villages are serviced by two major health centers (Mugil and Alexishafen) and several aid posts that are responsible for delivering EPI through monthly outreach clinics. Although ownership of bed nets was relatively high $(>50 \%)$, until the start of free long-lasting insecticide-treated net distribution by the national malaria program in late 2008, the majority of the population in the study site were using untreated nets.

Originally, the trial was started as a two-site study, with a second site in Wosera (East Sepik Province). However, an interim analysis performed mid-study found that the incidence of malaria was dramatically different between the two sites, restricting the comparability of the two sites. Consequently, enrollments were ceased in Wosera in May 2008 and, in line with a revised sample size calculation, enrollments at the Madang site were increased to 334 children per arm. The data from the Wosera cohort were subsequently used only for an overall safety analysis (Table S1).

The study was carried out in accordance with Good Clinical Practice (ICH GCP E6) guidelines and externally monitored by two independent monitors and the Data Safety Monitoring Board. The study was approved by the PNG Medical Research Advisory Committee (MRAC number 05.20). The trial was registered on http://www.clinicaltrials.gov (number NCT00285662) and formed part of the IPTi Consortium (http://www.ipti-malaria.org) [29].

\section{Randomization, Blinding, and Treatment Allocation}

Children were enrolled if they fulfilled the following criteria: (1) permanent resident of the area, (2) aged $3( \pm 1)$ mo old, (3) no disability, (4) no chronic illness, (5) no known allergy to study drugs, (6) $\mathrm{Hb}>5 \mathrm{~g} / \mathrm{dl}$, and (7) no severe malnutrition (defined by the PNG national guidelines as a weight less than $60 \%$ of the Harvard reference weight-for-age value [30]). After enrollment children were randomly allocated to the placebo, SP-AQ or SPAS treatment group using a pre-assigned list. Randomization lists were prepared by independent statistician using SAS software and consisted of assignments in blocks of 12, each comprising a list of the same 12 letters in random order. The three arms of the trial were each assigned four randomly selected letters from among the twelve, which were used to label the packaging of the tablets. The 
code list was held by the statistician in a locked drawer until completion of the trial. Individual allocation codes were contained in pre-prepared opaque envelopes, which were opened only after a child was allocated to the next available ID number.

$\mathrm{SP}, \mathrm{AS}, \mathrm{AQ}$ and their respective placebos were pre-packaged in individual blisters by the drug manufacturers (SP and AS, IPCA; AQ Kinapharma). Study drugs and placebo were identical in appearance and color, but as drugs were administered with sweet syrup no attempt was made to match the moderately bitter taste of AQ. The quality of all drugs and corresponding placebo was independently verified. Study drugs and placebo were assigned to the respective treatment codes by an independent technician. The entire study team and principal investigators remained fully blinded for the entire study period.

\section{Clinical Study Procedures}

The three different study interventions were (1) SP-AQ a single dose of SP $(25 \mathrm{mg} / \mathrm{kg}$ sulfadoxine and $1.25 \mathrm{mg} / \mathrm{kg}$ pyrimethamine) combined with $3 \mathrm{~d}$ of AQ $(10 \mathrm{mg} / \mathrm{kg})$ plus $3 \mathrm{~d}$ of $\mathrm{AS}$ placebo; (2) SP-AS, a single dose of SP plus $3 \mathrm{~d}$ of AS (4 mg/ $\mathrm{kg}$ ) plus $3 \mathrm{~d}$ of AQ placebo; (3) matching placebos for all three drugs. The tablets were split (quarters or halves) according to the weight (in kilograms) of each child. The two treatment arms corresponded to the first and second line treatments of the PNG national standard guidelines for malaria at that time.

The intervention was delivered four times during the first year of life alongside the EPI at 3, 6, 9, and 12 mo of age (Table 1). The drugs were crushed and mixed with water and sweet syrup (Golden Crush Cordial, Coca-Cola Amatil) for easy administration with spoon or syringe. The first day dose was administered by study staff. The children were monitored closely, and if they vomited within $30 \mathrm{~min}$ of receiving treatment, the dose was repeated. The second and third doses were given by the carer at home without direct supervision. Adherence and potential adverse reactions to the study drugs were assessed by community reporters, and an adherence rate of $>90 \%$ was reported (data not shown).

The parents or guardians of potential study participants were contacted during monthly routine EPI clinics run jointly by study staff and health center staff when the child was 1-2 mo of age. The study was explained in detail to the parents through both individual and community awareness meetings. An information brochure and consent form in English and in the local language (Pidgin) was given to the parents to take home for further discussion.

A child who met the inclusion criteria was formally enrolled during the next clinic visit, when the child was 3 mo of age $( \pm 1 \mathrm{mo})$ and after at least one parent gave written consent. Upon enrollment, a concise medical history including bed net use, possible disabilities, and presence of acute illness was performed. A brief physical examination was done, including measurement of weight. Table 1 summarizes what occurred at different visits during the trial.

Throughout the study period, a passive case detection system was maintained at the Mugil health center and three outlying clinics where study participants received treatment free of charge. Each illness episode was assessed by study staff using a standard case report form. In the case of history of fever within the past $48 \mathrm{~h}$ or an axillary temperature $>37.5^{\circ} \mathrm{C}$, a rapid malaria diagnostic test (ICT Combo) was performed, two blood smears and $250 \mathrm{ml}$ of whole blood were collected, and $\mathrm{Hb}$ level was measured using a portable HemoCue 201 machine. Only infants positive for malaria on the rapid diagnostic test were treated with artemether/ lumefantrine (Coartem). The children with moderate to severe anemia $(\mathrm{Hb}<8 \mathrm{~g} / \mathrm{dl})$ received iron supplementation (ferrous sulfate, $5 \mathrm{mg} / \mathrm{kg}$ daily for $6 \mathrm{wk}$ ). All other illnesses were treated according to the standard treatment guidelines of PNG. Children presenting with any danger signs or symptoms were referred to the health center for admission and treatment. Study participants who attended after hours were treated by health center staff based on presumptive diagnosis. In such cases, study staff recorded the reason for admission and (when possible) performed a finger prick to check parasitemia and $\mathrm{Hb}$ the next day.

All illness episodes were considered adverse events (AEs) and were graded by the study clinicians according to severity from grade 1 (less severe) to grade 3 or as a serious adverse event (SAE; life-threatening or deadly, resulting in disability/incapacity, or

Table 1. Summary of scheduled patient contacts during study follow-up.

\begin{tabular}{|c|c|c|c|c|c|c|c|c|}
\hline \multirow[t]{2}{*}{ Type of Visit } & \multicolumn{8}{|c|}{ Age (Months) } \\
\hline & 2 & 3 & 6 & 9 & 12 & 15 & 18 & 21 \\
\hline Pre-screening & $\mathrm{x}$ & & & & & & & \\
\hline IPTi treatment & & IPTi 1 & IPTi 2 & IPTi 3 & IPTi 4 & & & \\
\hline Primary efficacy assessment & & & & & & $\mathrm{x}$ & & \\
\hline Follow-up & & & & & & & $\mathrm{x}$ & \\
\hline Final assessment & & & & & & & & $\mathrm{x}$ \\
\hline Passive case detection & & $\mathrm{x}$ & $\mathrm{x}$ & $\mathrm{x}$ & $\mathrm{x}$ & $\mathrm{x}$ & $\mathrm{x}$ & $\mathrm{x}$ \\
\hline \multirow[t]{5}{*}{ Immunization } & OPV 3 & OPV 4 & & & & & & \\
\hline & & HBV 3 & & & & & & \\
\hline & DPT 2 & DPT 3 & & & & & & \\
\hline & & & Meas 1 & Meas 2 & & & & \\
\hline & & & Vit A & & Vit A & & & \\
\hline \multicolumn{9}{|l|}{ During schedules visits } \\
\hline Blood collection (finger prick) & & $500 \mu \mathrm{l}$ & $250 \mu \mathrm{l}$ & $250 \mu \mathrm{l}$ & $250 \mu \mathrm{l}$ & $1 \mathrm{ml}$ & $250 \mu \mathrm{l}$ & $250 \mu \mathrm{l}$ \\
\hline $\mathrm{Hb}$ levels & & $\mathrm{x}$ & & & & $\mathrm{x}$ & & \\
\hline
\end{tabular}

DTP, diphteria, pertussis, tetanus vaccine; HBV, hepatitis B vaccine; Meas, measles vaccine; OPV, oral polio vaccine; Vit A, oral vitamin A supplementation.

doi:10.1371/journal.pmed.1001195.t001 
presenting with specific clinical/laboratory features such as those that characterize important hematological disorders [as defined by the IPTi Consortium]). Hospital admissions that did not fulfill the criteria of SAE were considered grade 3. The study clinicians assessed all SAEs and causality of AEs. SAEs and AEs possibly or certainly related to the intervention were reported to the Data Safety Monitoring Board, which assessed grade and causality using IPTi Consortium guidelines.

\section{Diagnosis of Malaria Infections}

All blood slides were read by two expert microscopists. In case of discrepancies, a third read was performed. Thick blood films were examined by light microscopy for 200 thick-film fields (under $100 \times$ oil immersion lens) before being declared infection-negative. Slides were scored as light-microscopy-positive for an individual Plasmodium species, if the species was detected independently by at least two microscopists. Additionally, if the final slide readings were discrepant compared to PCR results (positivity and species), a final confirmatory read by a senior microscopist was performed. Parasite densities were recorded as the number of parasites per 200 white blood cells and converted to the number of parasites/ microliter assuming 8,000 white blood cells/microliter [3]. Final parasite densities were obtained by calculating the geometric mean of positive reads.

Blood samples were collected from finger pricks in tubes coated with ethylenediaminetetraacetic acid and/or in plain tubes (15-mo visit only). Upon arrival in the laboratory, samples were separated into plasma or serum and cell pellet. Plasma and serum were stored at $-80^{\circ} \mathrm{C}$ until further use. DNA was extracted from cell pellets using the QiaAMP 96 extraction kit (Qiagen) and the 96well Genomic DNA Extraction Kit (Favorgen).

The presence of each of the four human malarial species was assessed in all blood samples using a semi-quantitative post-PCR ligase detection reaction/fluorescent microsphere assay (LDRFMA) [31]. This assay combines PCR amplification of the small subunit ribosomal RNA gene (491- to 500-bp fragments) using genus-specific primers, followed by a multiplex species-specific ligation detection reaction. The design and sensitivity of this assay have been described previously [31,32]. The threshold of positivity of the reactions for each species was determined monthly using the mean value obtained for the negative controls (for each species), plus three times the value of its standard deviation. Samples with low-positive values ( $<$ [threshold $+8 \times$ standard deviation] $)$ were double-checked and considered as positive only if the result was confirmed.

\section{Sample Size Calculations}

Based on a predicted incidence rate of 1.2 episodes/child/year ( 0.7 for $P f$ and 0.5 for $P v$ ), a power of $90 \%$ and alpha $=0.025$, a sample size of 250 children per arm was estimated to be necessary to find at least a $28 \%$ reduction in the incidence of all malaria episodes, a $30 \%$ reduction in the incidence of $P v$, and a $35 \%-37 \%$ reduction in the incidence of $P f$ episodes. Assuming a drop out of participants of $20 \%$ in conjunction with a fall in incidence of malaria of $30 \%$ to $50 \%$ due to long-lasting insecticide-treated net introduction through the national program in November 2008, a final adjusted sample size of 366 children per arm, or 1,100 children in total, was calculated.

\section{Statistical Methods}

The analysis was performed both by modified intention to treat (mITT) and according to protocol (ATP). The mITT population includes all randomized children in Mugil that received at least one dose of IPTi or placebo (i.e., 1,121 of 1,125 children).
Following the mITT principle, participants were analyzed according to the preventive treatment they were assigned to at randomization. In the ATP analysis, the population includes all randomized participants who received at least three IPTi treatments (each given within $4 \mathrm{wk}$ after the scheduled treatment time point) and were under passive surveillance for the entire 12mo period.

The primary objective of the trial was to evaluate the protective effect of the two IPTi regimens. The primary outcome was measured as the incidence of symptomatic malaria due to any Plasmodium species from 3 to 15 mo of age. Secondary outcomes included (1) the specific incidence of symptomatic malaria due to $P f$ or $P v$ from 3 to 15 mo of age, (2) the incidence of symptomatic malaria (any, $P f$, and $P v$ ) from 15 to 21 mo (i.e., "rebound"), (3) the incidence of moderate-to-severe anemia $(\mathrm{Hb}<8 \mathrm{~g} / \mathrm{dl})$ and severe anemia $(\mathrm{Hb}<5 \mathrm{~g} / \mathrm{dl})$ from 3 to $15 \mathrm{mo}$ and 15 to $21 \mathrm{mo}$, and (4) the prevalence of malaria parasitemia at 15 and 21 mo.

Symptomatic malaria was defined as history of fever or axillary temperature $\geq 37.5^{\circ} \mathrm{C}$ and a positive blood smear or a positive rapid diagnostic test, confirmed by positive PCR. The incidences were compared between groups using negative binomial regression to take into account possible extra-Poisson variation due to frailty at the individual level. Analyses were adjusted by sex, number of IPTi treatments received, season of enrollment (wet versus dry), average bed net use, and site (grouped by 12 recruitment zones). The time at risk was calculated starting on the date of enrollment until the date defined according to the analysis, or until the participant was withdrawn from the study. An arbitrary period of $28 \mathrm{~d}$ was excluded after each malaria episode. The same methodology was used for evaluating of the incidence of moderate-to-severe $(\mathrm{Hb}<8 \mathrm{~g} / \mathrm{dl})$ and severe anemia $(\mathrm{Hb}<5 \mathrm{~g} /$ dl). Efficacy results are presented as either incidence rate ratio (IRR) or PE (PE = 1-IRR).

The risk of malaria within $35 \mathrm{~d}$ following the administration of each of the treatment doses was investigated by calculating incidence rates and hazard ratios using a Cox regression model. Differences in prevalence of infection at 15 and 21 mo were evaluated using logistic regression.

The analyses were performed in Stata version 11 (StataCorp).

\section{Results}

From 6 June 2006 to 14 May 2010, 1,125 3-mo-old infants were enrolled, randomized, and followed-up in the trial in the Mugil area of Madang Province (Figure 1). Four infants were retrospectively excluded from the analysis as they were already receiving antimalarial treatments at the time of all IPTi visits and therefore did not receive any IPTi treatment doses. In all, 374 infants were allocated to the SP-AQ group, 374 to the SP-AS group, and 373 to the placebo group (Figure 1). A total of 1,079 (96\%) participants completed follow-up to 15 mo, whereas 857 (76\%) completed the 6 mo post-treatment follow-up (i.e., 15-21 mo).

The baseline characteristics of all study infants were similar across the three treatment arms (Table 2). No significant imbalances were observed between the three groups. In the final multivariate models, gender, village of residence, average insecticide-treated net use, and season of recruitment were included. A total of 939 children who received three or four IPTi treatments were included in the ATP population, with no significant imbalances between groups (Table S2).

\section{Protective Efficacy}

The incidence of clinical malaria between the first dose at 3 mo and $15 \mathrm{mo}$ in the placebo arm was 1.07 per person-year at risk 

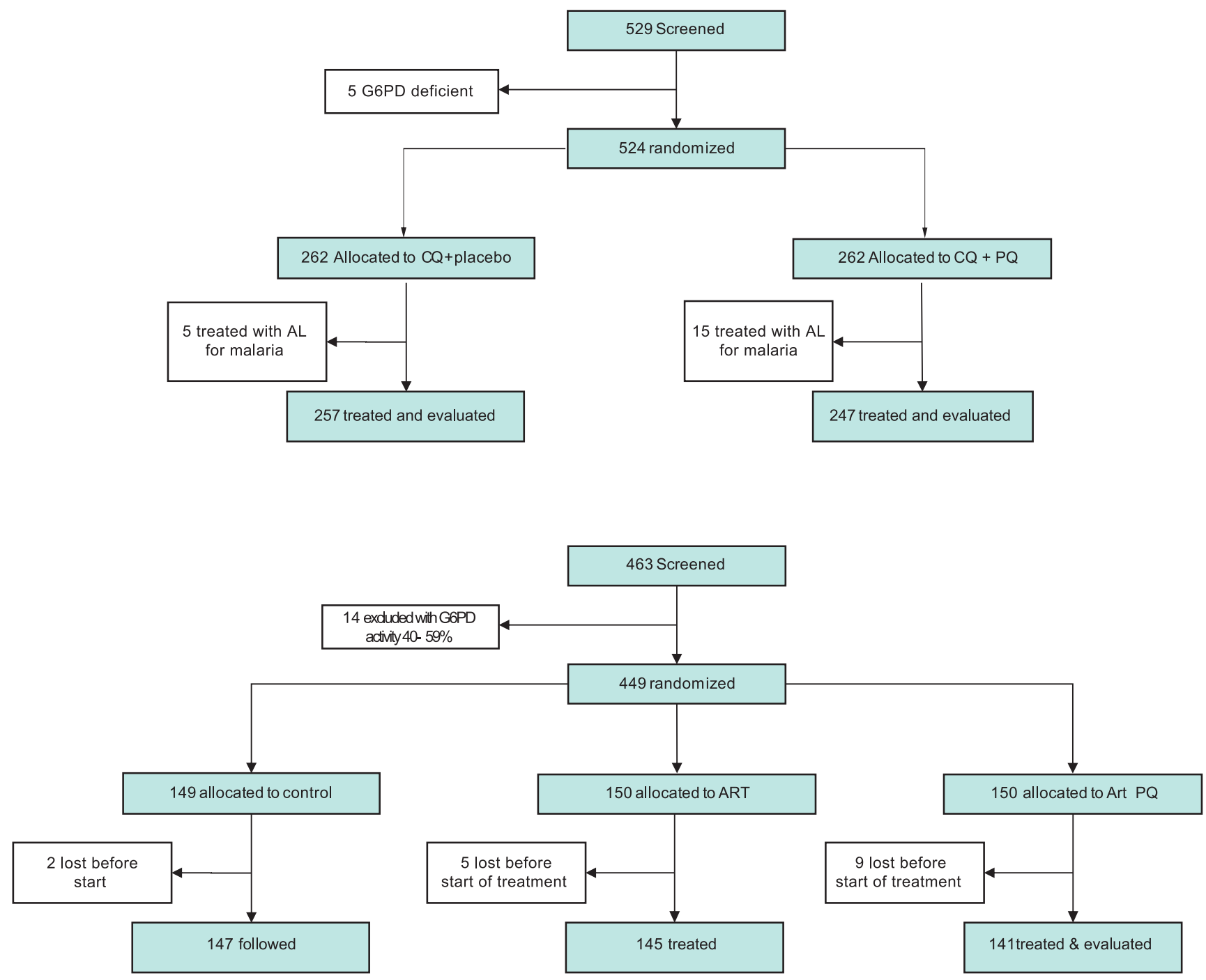

Figure 1. Trial profile. incomplete FU, incomplete follow-up (i.e., the study was terminated before the participant completed the 21-mo follow-up time period); LTF, lost to follow-up. doi:10.1371/journal.pmed.1001195.g001

(PYAR) overall, and 0.28 and 0.82 for $P f$ and $P v$, respectively. In mITT analyses, the incidence of all cases of malaria was $29 \%$ $(95 \%$ CI, $10-43, p \leq 0.001)$ lower in the SP-AQ group as compared to placebo, but not significantly reduced in the SP-AS group as compared to placebo (point estimate for change 12\%, 95\% CI, -11 to $30, p=0.12$ ). The $\mathrm{PE}$ was higher against $P f$ than $P v$ in both groups (Table 3). In the SP-AQ group, $P f$ incidence was reduced by $35 \%(95 \%$ CI, $9-54, p=0.012)$ and $P v$ incidence by $23 \%(95 \%$ CI, $0-41, p=0.048$ ) in comparison to placebo. In contrast, the SPAS group showed a significant reduction only for incidence of $P f$ (PE $=31 \%, 95 \%$ CI, 4-51, $p=0.027)$, not for incidence of $P v$ $(\mathrm{PE}=4 \%, 95 \% \mathrm{CI},-26$ to $24, p=0.759)$.

Estimates of protection were higher when only children with three or more IPTi treatments were considered (i.e., ATP analyses; Table 3). Regular administration of SP-AQ reduced malaria episodes by $38 \%$ overall (95\% CI, 20-52, $p<0.001)$, with reductions of $49 \%(95 \%$ CI, $26-65, p<0.001)$ and $31 \%(95 \%$ CI, 9-48, $p=0.010$ ) for $P f$ and $P v$ malaria episodes, respectively. Administration of SP-AS resulted in a $23 \%$ reduction in all malaria episodes $(95 \%$ CI, $1-40, p=0.041)$, with a statistically significant reduction for $P f(46 \%$ lower, 95\% CI, 22-62, $p=0.001$ ) but not for $P v$ (point estimate for change $12 \%, 95 \%$ CI, -16 to 34 , $p=0.355)$.

Comparable effects were seen when more specific definitions for $P f$ (i.e., parasite density $>2,500 / \mu \mathrm{l}$ ) and $P v$ (i.e., parasite density $>500 / \mu \mathrm{l}$ ) [33] were applied (Table 3). In the ATP cohort, in the SP-AQ arm only, a substantially higher protection was observed against higher density $\operatorname{Pf}(\mathrm{PE}=65 \%, 95 \%$ CI, 43-79, $p<0.001)$ but not against higher density $P v$. The results for the mITT cohort are comparable.

IPTi with SP-AQ or SP-AS also provided protection against moderate-to-severe anemia $(\mathrm{Hb}<8 \mathrm{~g} / \mathrm{dl}$; Table 3$)$. In the ATP population, the PE increased to $41 \%$ (95\% CI, 18-58, $p=0.002$ ) in the SP-AQ group and 34\% (95\% CI, $8-53, p=0.013)$ in the SPAS group. Few cases of severe anemia $(<5 \mathrm{~g} / \mathrm{dl})$ were observed during the study. In the ATP analysis, there were seven cases in the placebo group, one in the SP-AQ group, and two in the SP-AS group $(p=0.04)$. Results for the mITT population were of similar magnitude, although in general the estimates obtained were lower and not statistically significant $(p=0.09-0.13)$ (see Table 3). 
Table 2. Baseline characteristics of study participants-intention-to-treat cohort.

\begin{tabular}{|c|c|c|c|c|}
\hline Characteristic & Subcategory & Placebo & SP-AQ & SP-AS \\
\hline Total $n$ & & 373 & 374 & 374 \\
\hline Female & & $192(51.5 \%)$ & 166 (44.4\%) & $187(50 \%)$ \\
\hline \multirow[t]{12}{*}{ Village area } & Biranis & $18(4.8 \%)$ & $20(5.4 \%)$ & $18(4.8 \%)$ \\
\hline & Megiar & $20(5.4 \%)$ & $17(4.6 \%)$ & $22(5.9 \%)$ \\
\hline & Aronis Garup Wasabamal Zizzi & $41(10 \%)$ & 35 (9.4\%) & $42(11.2 \%)$ \\
\hline & Basken Dimer & 77 (20.6\%) & 74 (19.9\%) & $72(19.3 \%)$ \\
\hline & Bunu Kusen Mugil & $38(10.2 \%)$ & $54(14.4 \%)$ & $54(14.4 \%)$ \\
\hline & Matukar Wasab & $38(10.2 \%)$ & $31(8.3 \%)$ & $24(6.4 \%)$ \\
\hline & Dylup & $20(5.4 \%)$ & $29(7.8 \%)$ & $22(5.9 \%)$ \\
\hline & Karkum & $16(4.3 \%)$ & $8(2.1 \%)$ & $20(5.6 \%)$ \\
\hline & Mirap & $17(4.6 \%)$ & $23(6.2 \%)$ & $31(8.3 \%)$ \\
\hline & Sareng & $30(8 \%)$ & $21(5.6 \%)$ & $23(6.2 \%)$ \\
\hline & Taldig & $41(11 \%)$ & $48(12.8 \%)$ & $33(8.8 \%)$ \\
\hline & Rempi & $17(4.6 \%)$ & $14(3.7 \%)$ & $13(3.5 \%)$ \\
\hline Slept under bed net last $2 \mathbf{w k}^{\mathrm{a}}$ & & $308(82.8 \%)$ & $299(80 \%)$ & $305(82 \%)$ \\
\hline Recruitment during rainy season (October-June) ${ }^{a}$ & & $303(73.5 \%)$ & $301(73.0 \%)$ & $301(72.5 \%)$ \\
\hline Mean age at enrollment (days) ${ }^{a}$ & & $96(15)$ & $99(18)$ & $98(16)$ \\
\hline Weight (kilograms) ${ }^{a}$ & & $5.9(0.8)$ & $6.0(0.9)$ & $6.0(0.8)$ \\
\hline $\mathrm{Hb}$ (grams/decaliter) ${ }^{\mathrm{a}}$ & & $9.5(1.0)$ & $9.5(1.2)$ & $9.5(1.1)$ \\
\hline \multirow[t]{3}{*}{ Prevalence of parasitemia at baseline (light microscopy) } & All species & $14(3.8 \%)$ & $30(8 \%)$ & $24(6.4 \%)$ \\
\hline & Pf & $7(1.9 \%)$ & $13(3.5 \%)$ & $9(2.4 \%)$ \\
\hline & Pv & $7(1.9 \%)$ & $17(4.5 \%)$ & $15(4 \%)$ \\
\hline \multirow[t]{3}{*}{ Prevalence of parasitemia at baseline (PCR) } & All species & $69(18.5 \%)$ & $87(23.3 \%)$ & $75(20.1 \%)$ \\
\hline & $P f$ & $17(4.6 \%)$ & $20(5.4 \%)$ & $15(4 \%)$ \\
\hline & $P v$ & $52(13.9 \%)$ & $74(19.8 \%)$ & $63(16.8 \%)$ \\
\hline
\end{tabular}

Data are $n$ (percent) unless otherwise indicated.

${ }^{\text {a }}$ Data are mean (standard deviation).

doi:10.1371/journal.pmed.1001195.t002

Adjustment for gender, village of residency, season of recruitment, and use of bed nets did not significantly alter estimates of PE (Figure 2).

\section{Post- and Inter-Dose Efficacy}

The combined PE in the $35 \mathrm{~d}$ following the administration of each IPTi treatment dose showed a reduction of $80 \%$ (95\% CI, $67-88, p<0.001)$ with SP-AQ and 63\% (95\% CI, 45-76, $p<0.001)$ with SP-AS for all malaria infection (Table 3). SP-AQ had slightly lower PE against $P f(72 \%, 95 \%$ CI, 33-87, $p<0.001)$ than against Pv $(83 \%, 95 \%$ CI, 70-90, $p<0.001)$, whereas SP-AS was more efficacious against $P f(82 \%, 95 \%$ CI, $53-93, p<0.001)$ than against Pv $(59 \%, 95 \%$ CI, 47-73, $p<0.001)$. Both SP-AQ (PE $=43 \%, 95 \%$ CI, $13-63, p=0.010)$ and SP-AS (PE $=38 \%, 95 \%$ CI, 6-59, $p=0.026)$ were associated with a significant reduction in the incidence of $P f$ malaria between IPTi doses (i.e., the period from $35 \mathrm{~d}$ after a treatment dose to the next IPTi dose). However, no effect on the incidence of $P v$ malaria was observed for either treatment group beyond the first $35 \mathrm{~d}$ (Table 3).

\section{Safety}

A total of 6,165 AEs and 275 SAEs were observed during the intervention period, with no significant difference between treatment arms (Table 4). None of the SAEs were judged (by the Data Safety Monitoring Board and IPTi Safety Working
Group) to be intervention-related. One child in the placebo group experienced a non-severe (grade 1) skin rash a few days following administration of the second IPTi dose. Vomiting was reported in $2 \%$ of infants receiving SP-AQ $1.4 \%$ of infants receiving SP-AS, and $0.6 \%$ of infants receiving placebo $(p=0.001)$.

In total, ten deaths occurred among study participants. One was excluded from the analysis because he/she did not receive any IPTi treatment dose. Out of the nine study deaths, seven occurred during the intervention period (i.e., 3-15 mo of age). Of these, six occurred in the placebo group, one in the SP-AS group, and none in the SPAQ group $(p=0.011)$. An additional two deaths (one each in the SP$\mathrm{AS}$ and placebo groups) were observed during post-intervention follow-up. The likely diagnoses for the nine deaths were as follows: one severe $P v$ malaria (parasite density of $12,200 / \mu \mathrm{l}$ ) with severe anemia and possible lower respiratory tract infection (severe respiratory distress and cough), three lower respiratory tract infections, two severe dehydrations, one meningitis, and two unknown diagnoses.

\section{Post-Intervention Risk}

The incidence of clinical malaria between 15 and 21 mo was 1.09 PYAR overall and 0.43 and 0.70 for $P f$ and $P v$, respectively. No evidence of a "rebound effect" was observed in the 6 mo following the end of the intervention at the age of $15 \mathrm{mo}$. In both mITT and ATP populations, no significant difference in incidence of all malaria episodes was observed between treatment and 


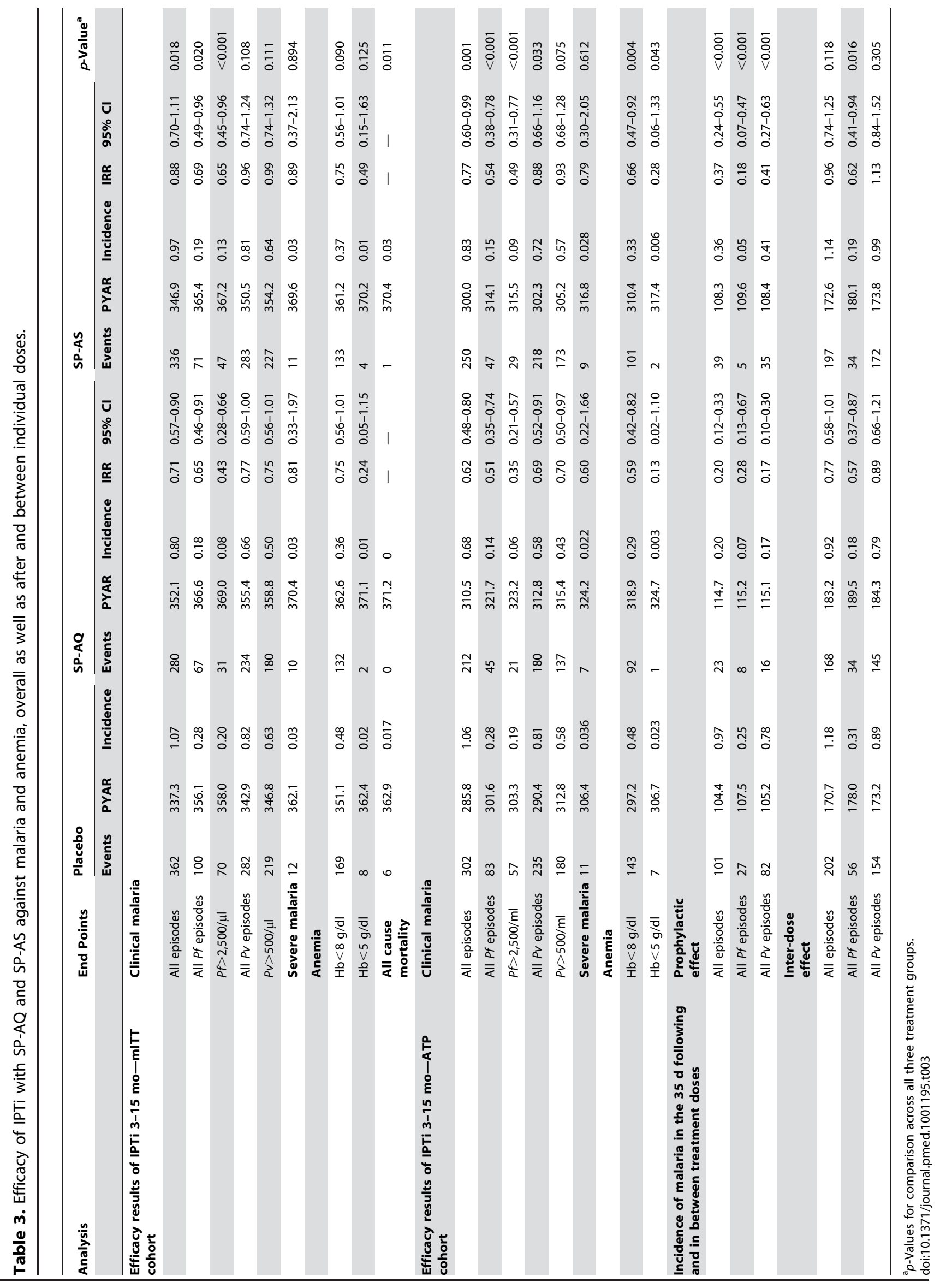




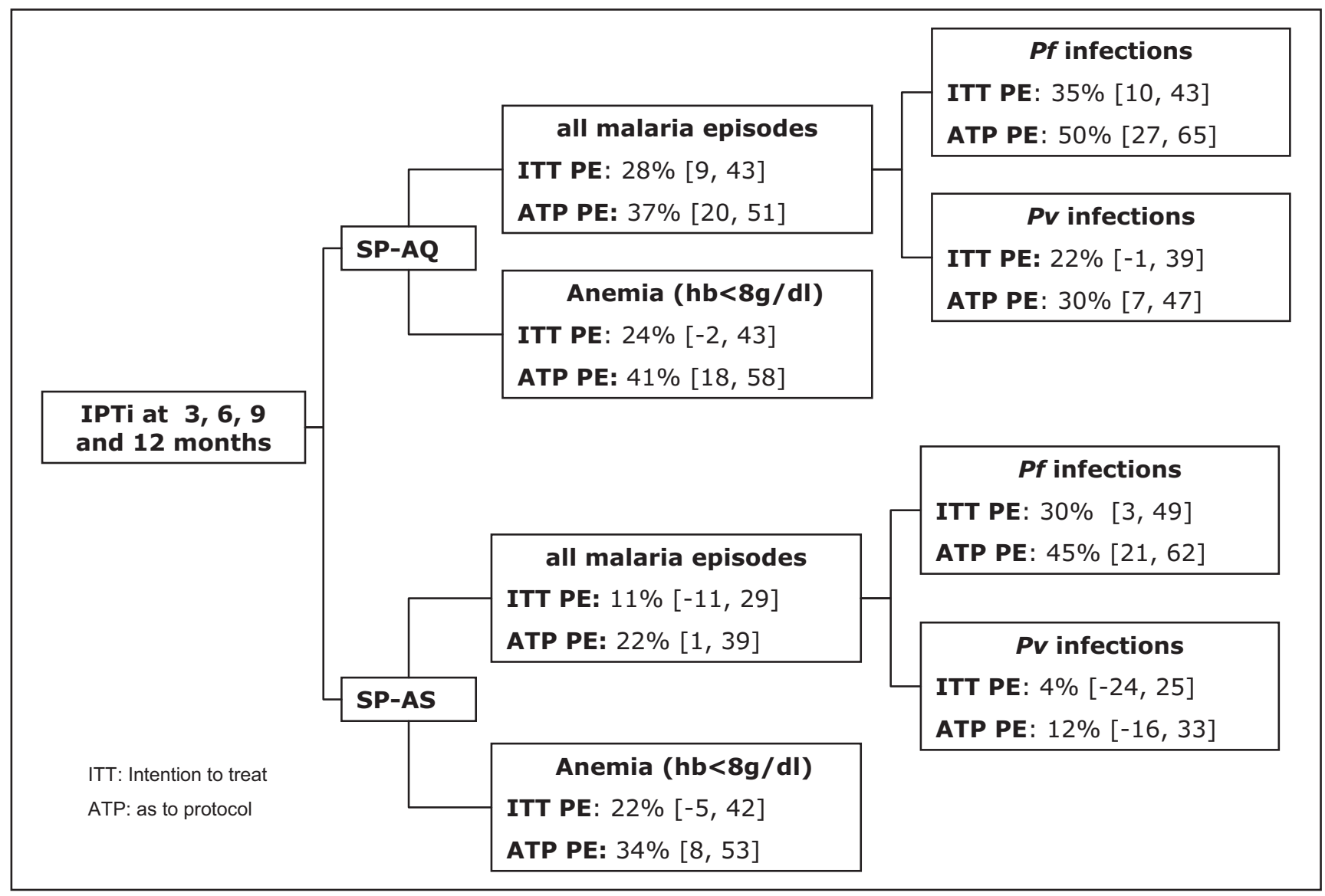

Figure 2. Summary of IPTi preventive efficacy against malaria at 15 mo of age adjusted for sex, place of residence, season of enrollment, and average insecticide-treated net use.

doi:10.1371/journal.pmed.1001195.g002

intervention groups (Table $4 ; p=0.56$ ). Similarly, no significant differences in incidence of $P f$ malaria, $P v$ malaria, and anemia were observed.

\section{Prevalence of Parasitemia during Follow-Up}

The prevalence rates of both $P f$ and $P v$ increased from $1.5 \%$ and $8.5 \%$ at 6 mo to $3.0 \%$ and $15.8 \%$ at 15 mo and $4.4 \%$ and $20.3 \%$ at $21 \mathrm{mo}$, respectively, when detected by microscopy (Figure 3). Using molecular diagnosis, prevalence of $P f$ and $P v$ was higher, and also increased with age (Figure 3). At $15 \mathrm{mo}$, the prevalence of $P f$ was significantly lower in the two treatment arms $(p=0.037)$ by light microscopy but not by LDR-FMA. No significant differences in prevalence were observed between treatment arms at 21 mo or any other time point for either $P f$ or $P v$. Infections with $P$. malariae and $P$. ovale were rare by microscopy (overall prevalence $P$. malariae: $0.1 \%, P$. ovale: $0.0 \%)$ and LDR-FMA (P. malariae: $1.0 \%, P$. ovale: $0.4 \%)$.

\section{Discussion}

This study in PNG infants provides the first evidence of which we are aware for the efficacy of IPTi with SP-based regimens for the prevention of malaria and anemia in a non-African population, including data on the efficacy against $P v$ malaria. IPTi with a combination of SP plus $3 \mathrm{~d}$ of AQ resulted in a $29 \%$ decrease in all malaria episodes and a $25 \%$ decrease in episodes of moderate-to-severe anemia. PE was higher in children who received at least three doses of IPTi $38 \%$ for all malaria and $41 \%$ for anemia). In line with local resistance patterns and SP-AS pharmacokinetic properties, SP-AS was found to be inferior to SPAQ. Overall, the efficacy of IPTi with SP-AQ in PNG infants is comparable to the $30 \%$ efficacy observed in other studies of African infants receiving IPTi with SP [10]. Importantly, SP-AQ has significant efficacy against both $P f$ and $P v$ malaria. Both IPTi treatments were safe and well tolerated. Therefore, these findings provide essential proof-of-principle evidence for the safety and efficacy of IPTi outside Africa, which has important implications for public health policy in non-African settings.

\section{Prevention of Malaria}

Overall, IPTi with SP-AQ was more effective in preventing $P f$ than Pv malaria (mITT $35 \%$ versus $23 \%$, ATP $49 \%$ versuss $31 \%$ ), and was more effective than SP-AS for $P f$ and $P v$ malaria. This was not unexpected. Two recent African clinical trials of IPTi have investigated alternative drug regimens, including at least one long half-life antimalarial such as mefloquine (125 mg single dose), a combination of SP (single dose) plus $3 \mathrm{~d}$ of $\mathrm{AS}$, or AQ plus AS. These treatments had protective efficacies ranging from $26 \%$ to $38 \%[16,34]$ and were comparable to those in earlier SP trials. However, a shorter acting combination, dapsone/chlorproguanil failed to significantly reduce the burden of malaria or anemia $[16,34]$. Similarly, in the earlier IPTi SP trials, the preventative effect of IPTi SP was almost entirely due to a reduction of malaria in the first $5 \mathrm{wk}$ after treatment [10,35]. Together, these results 
Table 4. Safety of IPTi with SP-AQ and SP-AS: adverse events and effect of incidence of malaria and anemia.

\begin{tabular}{|c|c|c|c|c|c|c|c|c|c|c|c|c|c|c|}
\hline \multirow{2}{*}{ Analyses and Endpoints } & \multicolumn{3}{|l|}{ Placebo } & \multicolumn{5}{|l|}{ SP-AQ } & \multicolumn{5}{|l|}{ SP-AS } & \multirow[t]{2}{*}{${ }_{\text {Value }}^{p}$} \\
\hline & Events & PYAR & Incidence & Events & PYAR & Incidence & $\mathbf{I R R}^{\mathbf{a}}$ & $95 \% \mathrm{Cl}$ & Events & PYAR & Incidence & $\mathbf{I R R}^{\mathbf{a}}$ & $95 \% \mathrm{Cl}$ & \\
\hline \multicolumn{15}{|l|}{$\begin{array}{l}\text { Safety of IPTi } \\
\text { 3-15 mo - mITT cohort }\end{array}$} \\
\hline All adverse events & 1,353 & 359.4 & 3.76 & 1,348 & 367.7 & 3.67 & 0.97 & $0.88-1.08$ & 1,353 & 366.8 & 3.69 & 0.98 & $0.88-1.09$ & 0.867 \\
\hline Serious adverse events & 65 & 362.8 & 0.18 & 83 & 371.0 & 0.22 & 1.22 & $0.83-1.79$ & 81 & 370.1 & 0.22 & 1.20 & $0.81-1.77$ & 0.550 \\
\hline \multicolumn{15}{|l|}{ Clinical malaria } \\
\hline All episodes & 171 & 149.0 & 1.15 & 164 & 155.5 & 1.05 & 0.91 & $0.70-1.20$ & 167 & 157.2 & 1.06 & 0.92 & $0.70-1.21$ & 0.763 \\
\hline All Pf episodes & 65 & 156.2 & 0.42 & 68 & 162.0 & 0.42 & 1 & $0.68-1.48$ & 74 & 163.8 & 0.45 & 1.08 & $0.74-1.58$ & 0.907 \\
\hline Pf $>2500 / \mu l$ & 41 & 157.8 & 0.26 & 43 & 163.6 & 0.26 & 1.01 & $0.63-1.62$ & 51 & 165.0 & 0.31 & 1.19 & $0.76-1.88$ & 0.693 \\
\hline All Pv episodes & 115 & 152.9 & 0.75 & 112 & 158.8 & 0.71 & 0.93 & $0.67-1.30$ & 106 & 161.1 & 0.66 & 0.88 & $0.63-1.23$ & 0.753 \\
\hline $\mathrm{Pv}>500 / \mu \mathrm{l}$ & 86 & 154.9 & 0.56 & 84 & 160.6 & 0.52 & 0.93 & $0.63-1.38$ & 78 & 162.9 & 0.48 & 0.87 & $0.58-1.29$ & 0.779 \\
\hline \multicolumn{15}{|l|}{ Anemia } \\
\hline $\begin{array}{l}\text { Moderate-to-severe } \\
(\mathrm{Hb}<8 \mathrm{~g} / \mathrm{dl})\end{array}$ & 42 & 157.7 & 0.27 & 44 & 163.4 & 0.27 & 1.01 & $0.63-1.61$ & 45 & 165.4 & 0.27 & 1.03 & $0.64-1.63$ & 0.994 \\
\hline \multicolumn{15}{|l|}{ Clinical malaria } \\
\hline All episodes & 140 & 127.4 & 1.10 & 138 & 136,2 & 1.01 & 0.91 & $0.67-1.24$ & 143 & 134.6 & 1.06 & 0.96 & $0.71-1.30$ & 0.833 \\
\hline All Pf episodes & 54 & 133.1 & 0.41 & 56 & 141.8 & 0.39 & 0.97 & $0.63-1.48$ & 63 & 140.1 & 0.45 & 1.10 & $0.73-1.67$ & 0.809 \\
\hline Pf $>2500 / \mu l$ & 34 & 134.5 & 0.25 & 35 & 143.2 & 0.24 & 0.96 & $0.57-1.62$ & 44 & 141.2 & 0.31 & 1.25 & $0.75-2.03$ & 0.560 \\
\hline All Pv episodes & 94 & 130.6 & 0.72 & 95 & 138.9 & 0.68 & 0.95 & $0.66-1.37$ & 91 & 137.9 & 0.66 & 0.92 & $0.64-1.34$ & 0.911 \\
\hline $\mathrm{Pv}>500 / \mu \mathrm{l}$ & 71 & 132.2 & 0.54 & 72 & 140.5 & 0.51 & 0.94 & $0.61-1.46$ & 68 & 139.4 & 0.49 & 0.92 & $0.59-1.43$ & 0.928 \\
\hline \multicolumn{15}{|l|}{ Anemia } \\
\hline $\begin{array}{l}\text { Moderate-to-severe } \\
(\mathrm{Hb}<8 \mathrm{~g} / \mathrm{dl})\end{array}$ & 40 & 134.1 & 0.30 & 37 & 142.9 & 0.26 & 0.87 & $0.43-1.44$ & 39 & 141.6 & 0.28 & 0.93 & $0.56-1.43$ & 0.862 \\
\hline
\end{tabular}

suggest that IPTi works mainly through a prophylactic effect, achieved by long-acting drugs preventing new infections, rather than by eradicating existing parasitemia [35]. Consistent with the African results, in the present study both SP-containing regimens had similarly high protective effect against $P f$ for the first $35 \mathrm{~d}$ $(72 \%-82 \%)$ as well as a moderate inter-dose effect, resulting in an overall efficacy of IPTi against $P f$ that was towards the higher end of those found in African studies [10,16,34].

SP-AQ also prevented $83 \%$ of Pv episodes in the first $35 \mathrm{~d}$ following treatment, indicating a similar post-treatment prophylactic effect, but the overall efficacy of IPTi against $P v$ malaria was lower than for Pf. SP-AS, however, showed significantly less posttreatment prophylactic effect $(\mathrm{PE}=59 \%)$ and no effect against $P v$ malaria overall. The most probable reasons for this are the ability of $P v$ to relapse from long-lasting liver stages [36] and higher levels of SP resistance in $P v$. Pv strains from New Guinea are thought to relapse very frequently [37], and $P v$ blood-stage infections are therefore re-established soon after drug treatment. In a concurrently conducted drug trial, $49 \%$ of PNG children $0.5-5$ y treated with SP-chloroquine $(3 \mathrm{~d})$ for $P v$ malaria had recurrent $P v$ parasitemia, and an additional $18 \%$ had a recurrent $P v$ clinical episode by day 42 , while $26 \%$ and $6 \%$ of children treated for $P f$ malaria had recurrent Pf parasitemia and clinical episodes, respectively [38].

The differential efficacy of IPTi with SP-AQ and SP-AS for the prevention of $P f$ and $P v$ malaria is consistent with the pharmacokinetic properties of the drugs used and previously observed drug resistance patterns. PCR-corrected day 28 in vivo failure rates from studies conducted concurrently or just prior to the trial were $10.3 \%$ for SP-AQ [39] and $10.0 \%$ for SP-AS [38] for Pf, and 3.6\% for SP-AQ [40] and $11.8 \%$ for SP-AS [41] for Pv. As SP has never been used as monotherapy in PNG, there are no estimates of SP in vivo resistance for either $P f$ or $P v$. However, molecular markers of SP resistance have been analyzed for both species in the study population. In $P f$, the quintuple $P f$ dihydrofolate reductase (pfdhfr) 51-59-108/Pf dihydropteroate synthase (pfdhps) 436547 mutant genotype is associated with a high degree of drug resistance [42], while in Pv, the 57L-58R-61M-117T pvdhfr quadruple mutation (irrespective of pvdhps mutations) has been associated with increased risk of treatment failure [43,44]. The high frequency of the quadruple pvdhfr mutation in PNG $(52 \%$ [41]) suggests that the clinical efficacy of SP for $P v$ may be significantly compromised. In contrast, the absence of quadruple/ quintuple $p f d h f r / p f d h p s$ [45] suggests that SP retains substantial efficacy against $P f$.

Although chloroquine-resistant $P v$ was first described in PNG [46], there is a significantly higher level of 4-aminoquinoline resistance present in $P f$ than in $P v$. By the early 1990s, greater than $35 \%$ of $P f$ cases showed RII and RIII resistance [47], and mutations underlying chloroquine and $\mathrm{AQ}$ resistance were shown to be highly prevalent [48]. In 2003/2004, SP-AQ was $100 \%$ clinically effective, but recurrent $P v$ parasitemia was observed in $12 \%$ of children treated with SP-AQ [39]. The pvdhfr 57L-58R$61 \mathrm{M}-117 \mathrm{~T} / P v$ multidrug resistance 1 (pvmdr1) $976 \mathrm{~F}$ quintuple mutant genotype has been associated with parasitological failure [43]. Although the pomdr1 976F mutation was present in $\sim 70 \%$ 


\section{P. falciparum}

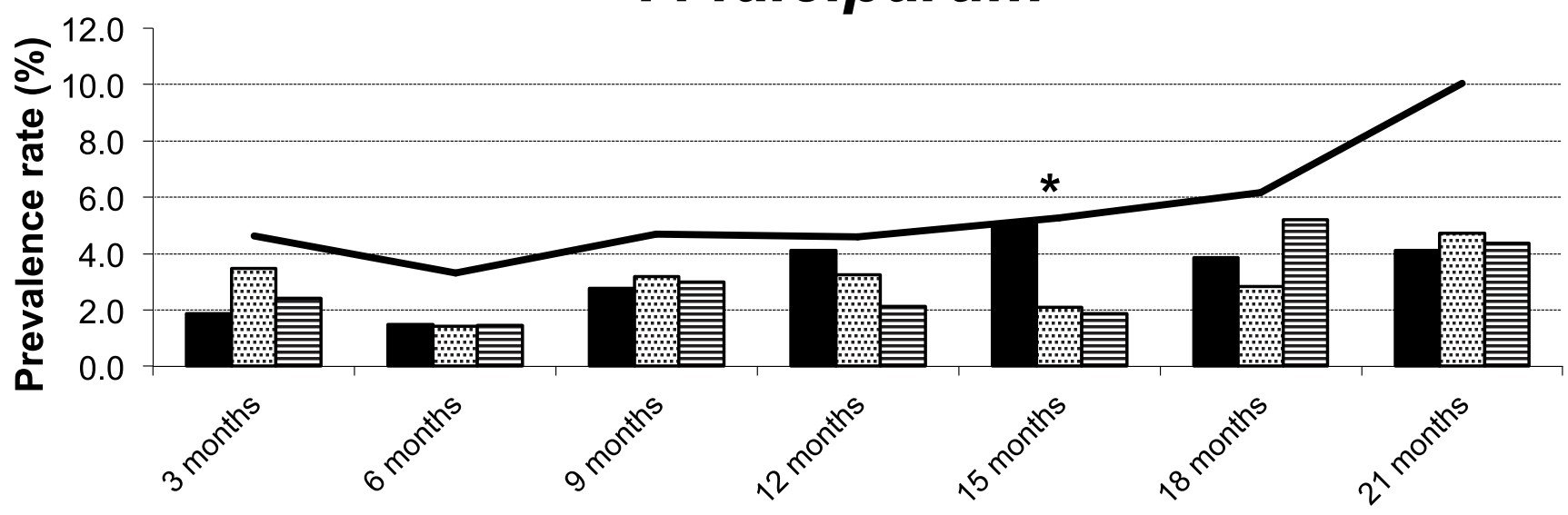

\section{P. vivax}

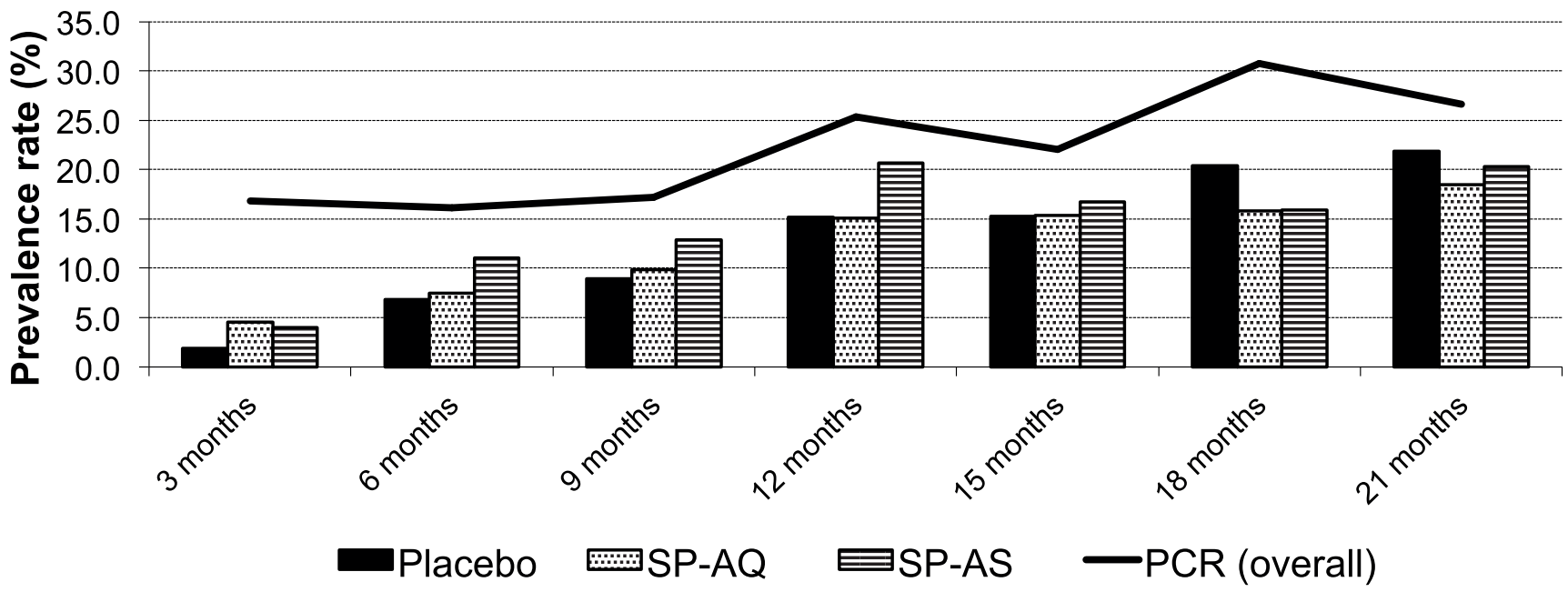

Figure 3. Prevalence of $P$. falciparum and $P$. vivax infections during follow-up by treatment group (light microscopy) and overall (PCR). * $p<0.05$.

doi:10.1371/journal.pmed.1001195.g003

of $P v$ isolates tested in 2006/2007, the predominantly late occurrence of recurrent parasitemia (i.e., day 28-42) following treatment with SP-chloroquine indicates that 4-aminoquinolines retained good clinical and at least partial parasitological efficacy [41].

Given the high level of AQ resistance and the very short halflife of AS [49], the efficacy of IPTi for the prevention of $P f$ is likely to be largely due to the effect of SP. The high level of preventative efficacy for $35 \mathrm{~d}$ is consistent with the terminal elimination half-life $\left(t_{1 / 2 \beta}\right)$ of 15.6 and $9.1 \mathrm{~d}$ for pyrimethamine and sulfadoxine, respectively, in PNG infants [50]. With SP having a greatly reduced efficacy against $P v$, the success of IPTi against $P v$ malaria relies largely on the efficacy of the partner drug. Whereas $P v$ parasites are efficiently cleared by SP-AS, the short half-life of AS provides no post-treatment prophylactic effect, and recurrent $P v$ infections are very common [38]. AQ on the other hand has a half-life of $\sim 9 \mathrm{~d}$ [51], and a 3-d course should therefore effectively suppress sensitive parasites effectively for 4-5 wk. The lack of any protective effect of SP-AS against $P v$ but not $P f$ adds further evidence to the observation that while SP can retain a considerable effect at low-to-moderate levels of resistance [14], it loses its prophylactic activity at high levels of resistance [16].

\section{Prevention of Anemia, Severe Illness, and Death}

Besides reducing the burden of malaria episodes, IPTi also reduced the risk of moderate-to-severe anemia (25\% in the mITT and $34 \%-41 \%$ in the ATP analyses, respectively) and severe anemia $(51 \%-87 \%)$. These effects are in line with previous findings on the prevention of anemia in Africa [10].

No difference was observed between the groups in number of SAEs (i.e., all cause admissions plus severe illness). Surprisingly, a significantly lower death rate was observed in the two treatment arms compared to the placebo arm. Even though this finding is very encouraging, it must be interpreted with caution. The present study was not powered to observe an effect of IPTi on mortality, and in the meta-analysis of six African studies, no effect of IPTi with SP on mortality was detected [10]. Only seven deaths occurred during the intervention period, with most children dying at home. Based on verbal autopsies and available clinical records, 
a majority of children might have died of severe lower respiratory tract infections and not from malaria.

\section{Safety of SP-AQ and SP-AS}

IPTi with SP-AQ or SP-AS was very safe. More than 4,000 doses of SP and 6,000 doses of $\mathrm{AQ}$ and $\mathrm{AS}$ were given, and no study-drug-related SAEs were observed, while AEs such as vomiting were rare in all groups. As reported in earlier studies, no significant increase in risk of malaria or anemia was recorded in the 6 mo after completion of the 12 mo of IPTi, indicating that IPTi does not impair the acquisition of immunity to both $P f$ and $P v$ and lead to a rebound in malaria risk. These data are very reassuring in regard to a possible use of these drug combinations in PNG and add to the safety data from African IPTi studies [10].

The use of SP as prophylaxis has been criticized because of the potential risk of Stevens-Johnson syndrome, a severe skin reaction [52], but by now $>15,000$ children have been treated with SPcontaining IPTi regimens in well-controlled clinical trials, and not a single drug-related severe dermatological event has been observed $[10,16,34,53]$. On the other hand, IPTi with mefloquine was highly effective, but was not well tolerated, with high rates of vomiting [16]. In the absence of novel drug regimens for IPTi, the combination of SP and AQ is the best currently available option for IPTi in PNG.

\section{Adherence, Acceptability, and Implementation}

The size of the difference in IPTi efficacy between the ATP and mITT analyses indicates that if the intervention is implemented, it will be important to achieve high coverage and high adherence in order to achieve good effectiveness.

In both the SP-AQ and SP-AS groups only the first dose was given as directly observed treatment. Parents were then counseled on the importance of giving the remaining two doses at home. With this approach a very high rate of compliance (>90\%) was achieved. As a result, SP and AQ day 4 drug levels were found to be comparable (N. Senn, P. Rarau, and I. Mueller, unpublished data) to those found in pharmacological studies with directly observed drug administration [50,54]. Acceptability of IPTi by parents and health workers of IPTi was excellent, and the administration of IPTi alongside EPI did not have negative impacts on attitudes to EPI, EPI adherence, or existing malaria prevention practices [55]. Therefore, and consistent with what was described in Africa [53], the IPTi intervention seems to be well suited for implementation in community settings in PNG, with high likelihood of both acceptability and adherence.

\section{Conclusion}

This study provides evidence of the efficacy of IPTi for the prevention of malaria and anemia in a region highly endemic for both $P f$ and $P v$. Additionally, this study provides an essential proof of principle that IPTi is an appropriate strategy for the prevention of $P v$ malaria, if an effective, long half-life drug is used. Policy makers should therefore consider IPTi in areas outside Africa or in countries in the Horn of Africa, where the burden of $P v$ malaria is high. Which areas are suited for IPTi introduction will depend on transmission level and choice of drug, both of which will affect the cost-effectiveness of the intervention [56]. While the results from Africa indicate that IPTi is a highly cost-effective intervention even

\section{References}

1. World Health Organization (2010) Guidelines for the treatment of malaria. Geneva: World Health Organization. at moderate levels of transmission [57], a formal evaluation of IPTi cost-effectiveness in non-African settings will be required to assist policy makers. Given the levels of resistance to SP and AQ in many parts of the Asia-Pacific and Americas, further studies are needed to investigate other combinations of long-acting drugs with a better efficacy against $P v$, in particular.

In the PNG context, the combination of SP-AQ (two longacting drugs with well-matched half-lives and good activity against either $P f[\mathrm{SP}]$ or $P v[\mathrm{AQ}]$ ) is an appropriate drug choice for IPTi, and its introduction into the national standard treatment guidelines should be considered. The replacement of $A Q$ and SP by artemether-lumefantrine as the national first line treatment will reduce the selection pressure for resistance against AQ and SP. Therefore, the efficacy of the two drugs in IPTi may be retained over time. Appropriate monitoring of prophylactic efficacy of AQ and SP, as well as evaluating new regimens, such as dihydroartemisinin-piperaquine, should accompany IPTi introduction in PNG.

\section{Supporting Information}

Table S1 Safety of IPTi with SP-AQ and SP-AS: AEs from both Madang and Maprik cohorts.

(DOCX)

Table S2 Baseline characteristics of study participants-ATP cohort.

(DOCX)

\section{Acknowledgments}

We are very grateful to the study participants, the populations, the members of community health associations, and the staff of the health centers of Mugil, Alexishafen, Kunjingini, and Kaugia for their cooperation throughout the study. Special thanks to the PNG IPTi field teams, in particular Joe Nale, Bridget Barber, the study nurses, and the community reporters for their dedicated work in the field; the microscopy, molecular parasitology, and immunology teams for accurate malaria diagnosis and sample preparation; Samson Akunaii, Carol Davy, Carol Taime, Lisa Kandi, Gilda Nongi, and the IPTi project management team for providing administrative and financial services support to the project; Rose Sabub for database management; Tom Smith (Swiss Tropical and Public Health Institute) for the production of the random allocation lists; and Tim Davis and team at the University of Western Australia for measurement of SP, AS, and AQ drug levels. Our special thanks go also to the members of the Data Safety Monitoring Board (John Vince, Trevor Duke, and Margaret Hellard), to the clinical monitors (Cornelia Bevilacqua and Leo Näscher), and to the PNG Department of Health and PNG Pediatric Society for their support and advice. Last but not least, we thank Kinapharma and IPCA, who manufactured the trial drugs.

\section{Author Contributions}

Conceived and designed the experiments: IM JCR SR PS LS JGB. Performed the experiments: NS PR DIS LR GB DM MS JI NT SL AR IM. Analyzed the data: JJA IM NS. Contributed reagents/materials/ analysis tools: PAZ. Wrote the first draft of the manuscript: IM NS PR. Contributed to the writing of the manuscript: JJA CB JCR SJR PAZ JGB LS PS LR DIS. ICMJE criteria for authorship read and met: NS PR DIS LR GB DM MS JI NT SL AR JJA PAZ JGB LS PS SJR JCR IM. Agree with manuscript results and conclusions: NS PR DIS LR CB DM MS JI NT SL AR JJA PAZ JGB LS PS SJR JCR IM. Enrolled patients: NS PR DM MS IM.

2. Lengeler C (2004) Insecticide-treated bed nets and curtains for preventing malaria. Cochrane Database Syst Rev 2004: CD000363. 
3. Pluess B, Tanser FC, Lengeler C, Sharp BL (2010) Indoor residual spraying for preventing malaria. Cochrane Database Syst Rev 2010: CD006657.

4. Sinclair D, Zani B, Donegan S, Olliaro P, Garner P (2009) Artemisinin-based combination therapy for treating uncomplicated malaria. Cochrane Database Syst Rev 2009: CD007483

5. Greenwood B (2004) The use of anti-malarial drugs to prevent malaria in the population of malaria-endemic areas. Am J Trop Med Hyg 70: 1-7.

6. Gosling R, Cairns M, Chico R, Chandramohan D (2010 May) Intermittent preventive treatment against malaria: an update. Expert Rev Anti Infect Ther 8: 589-606.

7. Menendez C, D'Alessandro U, ter Kuile FO (2007) Reducing the burden of malaria in pregnancy by preventive strategies. Lancet Infect Dis 7: 126-135.

8. Crawley J, Hill J, Yartey J, Robalo M, Serufilira A, et al. (2007) From evidence to action? Challenges to policy change and programme delivery for malaria in pregnancy. Lancet Infect Dis 7: 145-155.

9. Garner P, Gülmezoglu A (2006) Drugs for preventing malaria-related illness in pregnant women. Cochrane Database Syst Rev 2006: CD000169.

10. Aponte J, Schellenberg D, Egan A, Breckenridge A, Carneiro I, et al. (2009) Efficacy and safety of intermittent preventive treatment with sulfadoxinepyrimethamine for malaria in African infants: a pooled analysis of six randomised, placebo-controlled trials. Lancet 374: 1533-1542.

11. Meremikwu MM, Donegan S, Esu E (2008) Chemoprophylaxis and intermittent treatment for preventing malaria in children. Cochrane Database Syst Rev 2008: CD003756.

12. Institute of Medicine of the National Academies (2008) Assessment of the role of intermittent preventive treatment for malaria in infants: letter report. Washington (District of Columbia): Institute of Medicine of the National Academies.

13. Wilson AL, on behalf of the IPTc Taskforce (2011) A systematic review and meta-analysis of the efficacy and safety of intermittent preventive treatment of malaria in children (IPTc). PLoS ONE 6: e16976. doi:10.1371/journal. pone.0016976.

14. Griffin JT, Cairns M, Ghani AC, Roper C, Schellenberg D, et al. (2010) Protective efficacy of intermittent preventive treatment of malaria in infants (IPTi) using sulfadoxine-pyrimethamine and parasite resistance. PLoS ONE 5: e12618. doi:10.1371/journal.pone.0012618.

15. Mayor A, Serra-Casas E, Sanz S, Aponte JJ, Macete E, et al. (2008) Molecular markers of resistance to sulfadoxine-pyrimethamine during intermittent preventive treatment for malaria in Mozambican infants. J Infect Dis 197: $1737-1742$.

16. Gosling RD, Gesase S, Mosha JF, Carneiro I, Hashim R, et al. (2009) Protective efficacy and safety of three antimalarial regimens for intermittent preventive treatment for malaria in infants: a randomised, double-blind, placebo-controlled trial. Lancet 374: 1521-1532.

17. Gesase S, Gosling RD, Hashim R, Ord R, Naidoo I, et al. (2009) High resistance of Plasmodium falciparum to sulphadoxine/pyrimethamine in northern Tanzania and the emergence of dhps resistance mutation at codon 581. PLoS ONE 4: e4569. doi:10.1371/journal.pone.0004569.

18. World Health Organization (2010) WHO policy recommendation on intermittent preventive treatment during infancy with sulphadoxine-pyrimethamine (SPIPTi) for Plasmodium falciparum malaria control in Africa. Geneva: World Health Organization.

19. Rosenberg R (2007) Plasmodium vivax in Africa: hidden in plain sight? Trends Parasitol 23: 193-196.

20. Genton B, D'Acremont V, Rare L, Baea K, Reeder JC, et al. (2008) Plasmodium vivax and mixed infections are associated with severe malaria in children: a prospective cohort study from Papua New Guinea. PLoS Med 5: e127. doi:10.1371/journal.pmed.0050127.

21. Lin E, Kiniboro B, Gray L, Dobbie S, Robinson L, et al. (2010) Differential patterns of infection and disease with $\mathrm{P}$. falciparum and $\mathrm{P}$. vivax in young Papua New Guinean children. PLoS ONE 5: e9047. doi:10.1371/journal.pone.0009047.

22. Tjitra E, Anstey NM, Sugiarto P, Warikar N, Kenangalem E, et al. (2008) Multidrug-resistant Plasmodium vivax associated with severe and fatal malaria: a prospective study in Papua, Indonesia. PLoS Med 5: e128. doi:10.1371/journal. pmed.0050128.

23. Alexandre MA, Ferreira CO, Siqueira AM, Magalhaes BL, Mourao MP, et al. (2010) Severe Plasmodium vivax malaria, Brazilian Amazon. Emerg Infect Dis 16: $1611-1614$.

24. Lautze J, McCartney M, Krishen P, Olana D, Jayasinghe G, et al. (2007) Effect of a large dam on malaria risk: the Koka reservoir in Ethiopia. Trop Med Int Health 12: 982-989.

25. Mueller I, Galinski MR, Baird JK, Carlton JM, Kochar DK, et al. (2009) Key gaps in the knowledge of Plasmodium vivax, a neglected human malaria parasite. Lancet Infect Dis 9: 555-566.

26. Hawkins VN, Suzuki SM, Rungsihirunrat K, Hapuarachchi HC, Maestre A, et al. (2009) Assessment of the origins and spread of putative resistanceconferring mutations in Plasmodium vivax dihydropteroate synthase. Am J Trop Med Hyg 81: 348-355.

27. Cattani JA (1992) The epidemiology of malaria in Papua New Guinea. In: Attenborough R, Alpers M, eds. Human biology in Papua New Guinea: the small cosmos. Oxford: Clarendon Press. pp 302-312.

28. Schultz L, Wapling J, Mueller I, Ntsuke PO, Senn N, et al. (2010) Multilocus haplotypes reveal variable levels of diversity and population structure of
Plasmodium falciparum in Papua New Guinea, a region of intense perennial transmission. Malar J 9: 336

29. Schellenberg D, Cisse B, Menendez C (2006) The IPTi Consortium: research for policy and action. Trends Parasitol 22: 296-300.

30. Reed RB, Suart HC (1959) Patterns of growth in height and weight from birth to eighteen years of age. Pediatrics 24: 904-921.

31. McNamara DT, Thomson JM, Kasehagen LJ, Zimmerman PA (2004) Development of a multiplex PCR-ligase detection reaction assay for diagnosis of infection by four human malaria parasite species. J Clin Microbol 42: 2403-2410.

32. Kasehagen LJ, Mueller I, McNamara DT, Bockarie MJ, Kiniboro B, et al. (2006) Changing patterns of Plasmodium blood-stage infections in the Wosera region of Papua New Guinea monitored by light microscopy and high throughput PCR diagnosis. Am J Trop Med Hyg 75: 588-596.

33. Muller I, Genton B, Rare L, Kiniboro B, Kastens W, et al. (2009) Three different Plasmodium species show similar patterns of clinical tolerance of malaria infection. Malar J 8: 158.

34. Odhiambo FO, Hamel MJ, Williamson J, Lindblade K, ter Kuile FO, et al. (2010) Intermittent preventive treatment in infants for the prevention of malaria in rural western Kenya: a randomized, double-blind placebo-controlled trial. PLoS ONE 5: e10016. doi:10.1371/journal.pone.0010016.

35. May J, Adjei S, Busch W, Gabor J, Issifou S, et al. (2008) Therapeutic and prophylactic effect of intermittent preventive anti-malarial treatment in infants (IPTi) from Ghana and Gabon. Malar J 7: 198.

36. Krotoski WA (1989) The hypnozoite and malarial relapse. Prog Clin Parasitol 1: $1-19$.

37. Craige B, Alving AS, Jones R, Merrill Whorton C, Pullman TN, et al. (1947) The Chesson strain of Plasmodium vivax malaria: II. Relationship between prepatent period, latent period and relapse rate. J Infect Dis 80: 228-236.

38. Karunajeewa HA, Mueller I, Senn M, Lin E, Law I, et al. (2008) A trial of combination antimalarial therapies in children from Papua New Guinea. N Engl J Med 359: 2545-2557.

39. Marfurt J, Mueller I, Sie A, Maku P, Goroti M, et al. (2007) Low efficacy of amodiaquine or chloroquine plus sulfadoxine-pyrimethamine against Plasmodium falciparum and P. vivax malaria in Papua New Guinea. Am J Trop Med Hyg 77: 947-954

40. Koepfli C, Mueller I, Marfurt J, Goroti M, Sie A, et al. (2009) Evaluation of Plasmodium vivax genotyping markers for molecular monitoring in clinical trials. J Infect Dis 199: 1074-1080.

41. Barnadas C, Koepfli C, Karunajeewa HA, Siba PM, Davis TME, et al. (2011) Characterization of treatment failure in efficacy trials of drugs against Plasmodium vivax by genotyping neutral and drug resistance-associated markers. Antimicrob Agents Chemother 55: 4479-4481.

42. Picot S, Olliaro P, de Monbrison F, Bienvenu A, Price RN, et al. (2009) A systematic review and meta-analysis of evidence for correlation between molecular markers of parasite resistance and treatment outcome in falciparum malaria. Malaria J 8: 89.

43. Marfurt J, de Monbrison F, Brega S, Barbollat L, Muller I, et al. (2008) Molecular markers of in vivo Plasmodium vivax resistance to amodiaquine plus sulfadoxine-pyrimethamine: mutations in pvdhfr and pvmdr1. J Infect Dis 198: $409-417$.

44. Hawkins VN, Joshi H, Rungsihirunrat K, Na-Bangchang K, Sibley CH (2007) Antifolates can have a role in the treatment of Plasmodium vivax. Trends Parasitol 23: 213-222.

45. Wong RP, Karunajeewa H, Mueller I, Siba P, Zimmerman PA, et al. (2011) Molecular assessment of Plasmodium falciparum resistance to antimalarial drugs in Papua New Guinea using an extended ligase detection reaction fluorescent microsphere assay. Antimicrob Agents Chemother 55: 798-805.

46. Rieckmann KH, Davis DR, Hutton DC (1989) Plasmodium vivax resistance to chloroquine? Lancet 2: 1183-1184.

47. Muller I, Bockarie M, Alpers M, Smith T (2003) The epidemiology of malaria in Papua New Guinea. Trends Parasitol 19: 253-259.

48. Mehlotra RK, Fujioka H, Roepe PD, Janneh O, Ursos LM, et al. (2001) Evolution of a unique Plasmodium falciparum chloroquine-resistance phenotype in association with pfcrt polymorphism in Papua New Guinea and South America. Proc Natl Acad Sci U S A 98: 12689-12694.

49. Karunajeewa HA, Ilett KF, Dufall K, Kemiki A, Bockarie M, et al. (2004) Disposition of artesunate and dihydroartemisinin after administration of artesunate suppositories in children from Papua New Guinea with uncomplicated malaria. Antimicrob Agents Chemother 48: 2966-2972.

50. Salman S, Kose K, Griffin S, Baiwog F, Winmai J, et al. (2011) The pharmacokinetic properties of standard and double-dose sulfadoxine-pyrimethamine in infants. Antimicrob Agents Chemother 55: 1693-1700.

51. Stepniewska K, Taylor W, Sirima SB, Ouedraogo EB, Ouedraogo A, et al. (2009) Population pharmacokinetics of artesunate and amodiaquine in African children. Malar J 8: 200.

52. White NJ (2005) Intermittent presumptive treatment for malaria. PLoS Med 2: e3. doi:10.1371/journal.pmed.0020003.

53. Armstrong Schellenberg JRM, Shirima K, Maokola W, Manzi F, Mrisho M, et al. (2010) Community effectiveness of intermittent preventive treatment for infants (IPTi) in rural southern Tanzania. Am J Trop Med Hyg 82: 772-781.

54. Mwesigwa J, Parikh S, McGee B, German P, Drysdale T, et al. (2010) Pharmacokinetics of artemether-lumefantrine and artesunate-amodiaquine in children in Kampala, Uganda. Antimicrob Agents Chemother 54: 52-59. 
55. Pell C, Straus L, Phuanukoonnon S, Lupiwa S, Mueller I, et al. (2010) Community response to intermittent preventive treatment of malaria in infants (IPTi) in Papua New Guinea. Malar J 9: 369.

56. Ross A, Maire N, Sicuri E, Smith T, Conteh L (2011) Determinants of the costeffectiveness of intermittent preventive treatment for malaria in infants and children. PLoS ONE 6: e18391. doi:10.1371/journal.pone.0018391.
57. Conteh L, Sicuri E, Manzi F, Hutton G, Obonyo B, et al. (2010) The costeffectiveness of intermittent preventive treatment for malaria in infants in subSaharan Africa. PLoS ONE 5: e10313. doi:10.1371/journal.pone.0010313. 


\section{Editors' Summary}

Background Malaria is a major global public health problem. Half the world's population is at risk of this parasitic disease, which kills about one million people (mainly young children in sub-Saharan Africa) every year. Most of these deaths are caused by Plasmodium falciparum but $P$. vivax, the commonest and most widely distributed malaria parasite, is a major cause of malaria-related morbidity (illness and death) in many of the tropical and subtropical regions of the world where malaria is endemic (always present). Malaria is transmitted to people through the bites of night-flying mosquitoes. It can be prevented by controlling the mosquitoes that spread the parasite and by sleeping under insecticidetreated nets to avoid mosquito bites. Prompt treatment of malaria with antimalarial drugs can also reduce malaria transmission. In addition, intermittent preventative treatment (IPT) - the treatment of symptom-free individuals with full therapeutic courses of antimalarial drugs at fixed intervals regardless of their infection status-has been shown to reduce malaria-related morbidity among pregnant women in malaria-endemic areas and among African infants living in areas of high $P$. falciparum transmission.

Why Was This Study Done? The World Health Organization recently recommended that, in Africa, IPT should be given during infancy (called IPTi) at the same time as routine immunizations. Because the studies on which this recommendation is based were all carried out in subSaharan Africa, in populations where $P$. falciparum is the predominant parasite and $P$. vivax is uncommon, it is not known whether IPTi would be an appropriate prevention strategy in non-African settings or in regions where both $P$. falciparum and $P$. vivax are endemic. In this randomized placebo-controlled trial, the researchers investigate the efficacy of IPTi in infants living in an area of Papua New Guinea where $P$. falciparum and $P$. vivax are both highly endemic. In a randomized placebo-controlled trial, the effects of an intervention and of a placebo (dummy) intervention are compared in groups of individuals chosen through the play of chance.

What Did the Researchers Do and Find? The researchers assigned more than 1,000 infants to receive sulfadoxine/ pyrimethamine (SP) plus amodiaquine (AQ) (SP and $A Q$ are long-lasting antimalarial drugs), SP plus artesunate (AS) (AS is a short-lasting antimalarial), or placebo at 3,6, 9, and 12 months old. They recorded the number of malaria episodes that occurred among the children between the ages of 3 and 15 months. Then, by comparing the number of episodes occurring among the children receiving SP-AS or SP-AQ with the number occurring among the children receiving placebo, the researchers calculated the protective efficacy of the two drug combinations over the study period. The protective efficacy of IPTi against all clinical malaria episodes ( $P$. falciparum and $P$. vivax combined) was $29 \%$ for SP-AQ, but SP-AS was not associated with a statistically significant reduction in all malaria episodes as compared to placebo. For the two species of malaria separately, the incidence of $P$. falciparum malaria was $35 \%$ lower among the children receiving SP-AQ than among the children receiving placebo, whereas the incidence of $P$. vivax was reduced by $23 \%$; IPTi with SP-AS provided protection only against $P$. falciparum malaria (protective efficacy $31 \%$ ). Importantly, the number of adverse events (possible drug side effects) was similar in all the treatment arms, none of the severe adverse events were treatment-related, and there was no rebound in malariarelated morbidity for six months following the end of the intervention.

What Do These Findings Mean? These findings show that IPTi using a combination of long-lasting antimalarial drugs (SP-AQ) can effectively and safely prevent malaria in a nonAfrican population living in a region where $P$. falciparum and $P$. vivax are both highly endemic. Importantly, they also show that IPTi with SP-AQ can prevent both $P$. falciparum and $P$. vivax malaria. For Papua New Guinea, these findings suggest that SP-AQ is an appropriate drug choice for IPTi, particularly since the replacement of SP-AQ by artemether-lumefantrine as the national first line treatment for malaria will reduce the selection pressure for resistance against $\mathrm{SP}$ and $\mathrm{AQ}$. However, although these finding provide proof-of-principle evidence for the efficacy and safety of IPTi, further studies are needed to identify the most effective combinations of long-lasting antimalarial drugs for use in IPTi in other malaria-endemic regions.

Additional Information Please access these web sites via the online version of this summary at http://dx.doi.org/10. 1371/journal.pmed.1001195.

- Information is available from the World Health Organization on malaria (in several languages); the 2011 World Malaria Report provides details of the current global malaria situation; and the WHO policy recommendation on IPTi for P. falciparum malaria control in Africa is available

- The US Centers for Disease Control and Prevention provide information on malaria (in English and Spanish), including a selection of personal stories about malaria

- Information is available from the Roll Back Malaria Partnership on the global control of malaria, including a fact sheet about malaria in children and information on malaria in Papua New Guinea

- The IPTi Consortium was established to evaluate IPTi and inform public health policy making

- The Malaria Vaccine Initiative has a fact sheet on $P$. vivax malaria

- Vivaxmalaria.com provides information about $P$. vivax

- MedlinePlus provides links to additional information on malaria (in English and Spanish) 